Journal of Computer Science 6 (8): 928-933, 2010

ISSN 1549-3636

(C) 2010 Science Publications

\title{
Underactuated Anthropomorphic Finger Mechanism for Grasping and Pinching with Optimized Parameter
}

\author{
Norsinnira Zainul Azlan and Yamaura Hiroshi \\ Department of Mechanical and Control Engineering, Tokyo Institute of Technology, \\ Ookayama 2-12-1- 3-1, Meguro-Ku, Tokyo 152-8552, Japan
}

\begin{abstract}
Problem statement: Underactuated finger mechanism is beneficial in the anthropomorphic applications in which it reduces the finger size, weight and power consumption due to the less number of actuators compared to its number of Degree Of Freedom (DOF). However, the currently existing underactuated finger is limited to grasping operations only and unable to pinch with the finger tip. Moreover, the behavior of such finger in achieving the desired configuration depends greatly on its geometric parameters. Therefore, this study presented a new bar linkage type underactuated finger mechanism with an optimized parameter set that resembled the human finger in performing both pinching and grasping operation. Approach: The existing seven bar linkage underactuated finger mechanism was modified by adding a guiding slot in the upper middle phalange link so that its length can be adjusted to achieve human finger's configuration during pinching and grasping. A multiobjective optimization problem subjected to parameter constraints was then formulated using the evaluation criteria for human finger kinematics during pinching and grasping. Results: The numerical result obtained gave an improved set of geometrical parameter to achieve these configurations as it provided less error than the initial guess. Conclusion: A new underactuated finger mechanism that resembled the human finger to perform both pinching and grasping operation was presented in this study. The hardware fabrication will be carried out in the future using the optimized lengths obtained.
\end{abstract}

Key words: Underactuated finger, grasping, pinching, optimization

\section{INTRODUCTION}

Anthropomorphic hand finds its application in humanoid robots and prosthetic hands. Humanoid robot with anthropomorphic hand looks more natural while the replacement of an artificial hand that resembles the human hand helps the amputees who lost theirs due to accidents or health reasons to carry out their daily tasks. To be more specific, the term "anthropomorphism" refers to the capability of the robotic end effectors to mimic human's hand in terms of shape, size and weight. Over the last decades, numerous researches have been conducted to investigate the practical solution to achieve a balance between good physical appearance and functionality of such hand. Some of the examples of hand with high functionality include the UTAH/MIT Hand (Jacobsen et al., 1986) and the Stanford/JPL Hand (Salisbury and Craig, 1982). Although these hands are able to grasp and manipulate objects smoothly, their designs are bulky and complex due to high number of actuators and sensors. Another design approach adopts the design with lower number of actuators such as in Belgrade/USC hand (Bekey et al., 1990). Although it has successfully reduce the hand's weight and size, this method also decreases the number of Degree Of Freedom (DOF) of the hand, thus affects its versatility.

One of the simple techniques that maintain the humanistic appearance, size and weight of an artificial hand without scarifying its DOF is by adopting the underactuated mechanism approach. There are two main strategies in employing underactuated mechanism, which are by tendon or bar linkage. The tendon transmission is limited to small grasping forces whereas the link transmission is more suitable for applications in which large grasping forces are required. In both mechanisms, electric motors are replaced by passive elements such as spring and mechanical limits. Both of these mechanisms also result in self adaptive grasp and avoid the need of sensors and feedback control strategy.

However, most of the previous research on underactuated mechanism is limited to grasping function only such as in (Laliberte and Gosselin, 2001) and (Zollo et al., 2007). In reality, human finger can

Corresponding Author: Norsinnira Zainul Azlan, Department of Mechanical and Control Engineering,

Tokyo Institute of Technology, Ookayama 2-12-1-I3-1, Meguro-Ku, 152-8552, Japan 
also pinch with the fingertip to hold an object such as while holding a pencil during writing or to handle other small and thin items.

This study proposes a new mechanism of the underactuated hand to perform both grasping and pinching operation. The mechanism is based on bar linkage mechanism to provide higher grasping and pinching force. The upper link of the middle phalange is designed with a guiding slot so that the mechanism can adjust the length between Proximal Interphalangeal (PIP) and Distal Interphalangeal (DIP) joints so that it can achieve human configuration in these two positions. Further optimization of the design's geometric parameter is carried out to achieve an optimal solution with less error. The formulation is made based on kinematics equation of the four bar linkages and compared to human's finger configuration while performing these two operations.

\section{MATERIALS AND METHODS}

Review of human finger physiology: A normal human finger consists of finger bones called phalanges. The thumb has two phalanges and all the other four fingers consist of three phalanges. The farthest finger bone from the palm or below the nail is called distal phalanx. The proximal phalanx is the closest finger bone to the palm and the middle phalanx is located between the distal phalanx and the proximal phalanx. The middle and proximal phalanges are joined by Proximal Interphalangeal joint (PIP). The Metacarpal Phalangeal joint (MP) connects the proximal phalanx and the palm and the last joint in the finger, which is called Distal Interphalangeal joint (DIP) joins the distal and middle phalanges. The position of these phalanges and joints are illustrated in Fig. 1.

All of these phalanges have different configurations depending on the hand operation. There are two main finger operations studied in this research, which are grasping and pinching. Grasping operation is needed in power grasp, while pinching operation is utilized in manipulation or precision grasp. While grasping, all of the three joints in the right hand finger turn counterclockwise to hold an object, whereas in the pinching operation, only the MP and PIP joints turn counterclockwise and the DIP joint rotates in clockwise direction as illustrated in Fig. 2.

Artificial robotic finger mechanism and operation: The artificial anthropomorphic finger is designed so that it meets the following criteria:
- Resembles the human index finger in terms of physical appearance

- Resembles the human index finger in terms of size

- Resembles the human index finger in terms of weight

- Resembles the human index finger in terms of grasping and pinching functionality

To fulfill the first three criteria, the underactuated mechanism is chosen for this design. In this type of mechanism, the traditional actuators such as electromagnetic motors are replaced with small passive elastic elements and mechanical stops. This avoids the need of incorporating bulky actuators and sensors in the phalanges thus leading to the reduction of the finger size and weight without sacrificing the system's number of Degree Of Freedom (DOF).

To satisfy the last design requirement, the basic concept of underactuated SARAH's finger mechanism (Laliberte and Gosselin, 2001) is extended in this study. The DIP joint is designed to be able to turn counterclockwise to accomplish grasping configuration and turn clockwise to realize pinching motion.

The designed finger has three phalanges as human's and consists of MP, PIP and DIP joints that are responsible for the flexion and extension of the finger.

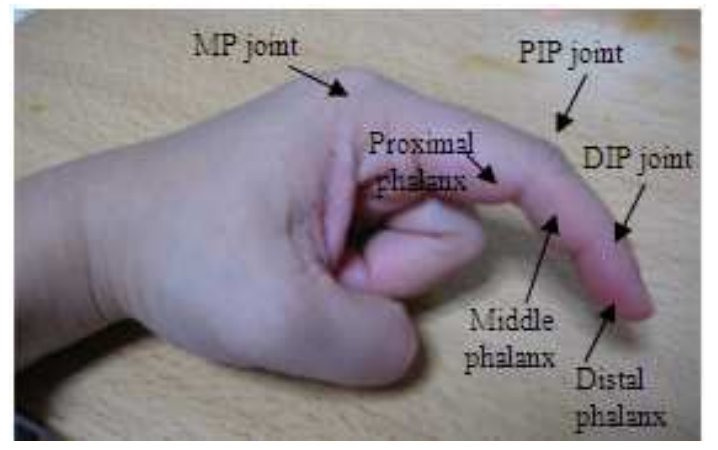

Fig. 1: Human finger's phalanges

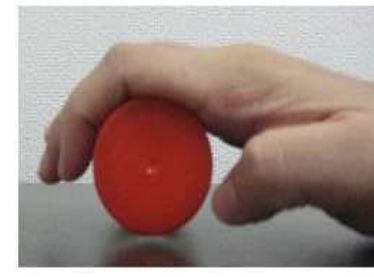

(a)

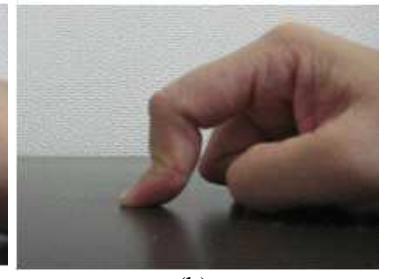

(b)
Fig. 2: Human finger's configuration during (a) grasping (b) pinching 


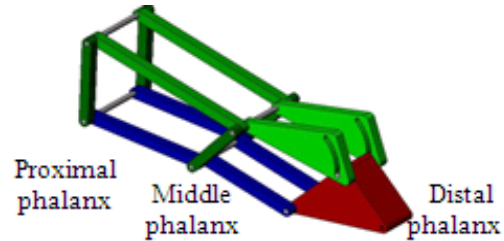

Fig. 3: Designed finger mechanism

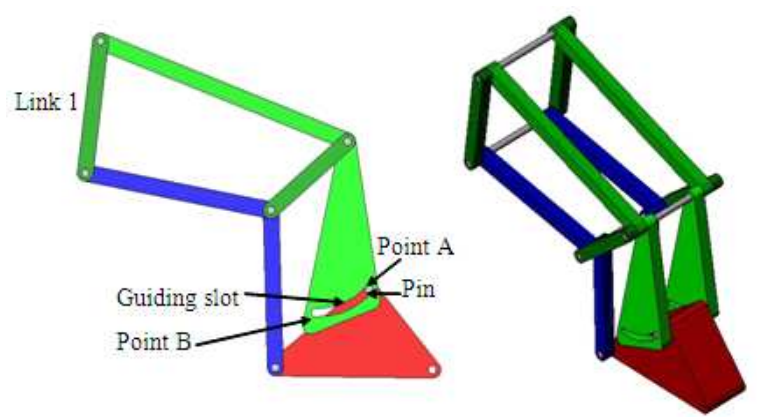

Fig. 4: Final finger mechanism configuration in pinching operation

It is made from seven linkage bar mechanism with the distal phalanx is constructed from a triangular bar as illustrated in Fig. 3. Only one input torque from the actuator is provided at the first link and the rest of the linkages move based on the contact made with the object. If the distal phalanx touches the object first, the finger will be in pinching mode, whereas if the proximal phalanx makes the first contact with the object, the finger will be in grasping operation. Since the upper link of the middle phalange need to be short in pinching operation and relatively long to realize grasping configuration, the upper link of this bar is modified by constructing it with a guiding slot. The pin in the distal phalanx moves along this slot depending whether the finger is in grasping or pinching operation.

In pinching operation, initially the finger behaves as one unit. Its original configuration is maintained by the spring in each joint. When an input torque is provided at Link 1, the distal phalange touches the ground or object and causes its pin to move along the guiding slot to point $\mathrm{A}$ on the upper link of the middle phalange. This shortens the length between point $\mathrm{C}$ and the pin of distal phalanx. Further bending of Link 1 leads the finger to achieve the final pinching configuration as shown in Fig. 4.

In grasping operation, similarly, the finger also behaves as a single body in the beginning. When the input torque is applied on Link 1, the entire finger rotates around the MP joint. This continues until one of the phalanges touches the object. When this happens, the phalanx in contact and its predecessors will stop to move.

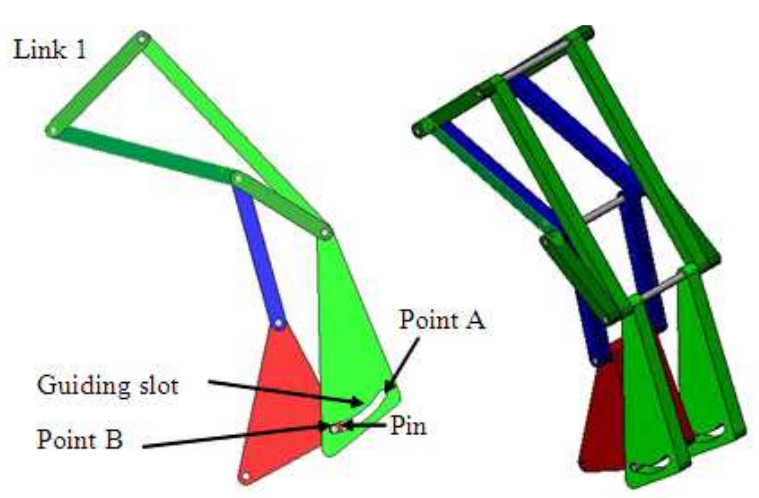

Fig. 5: Final finger mechanism configuration in grasping operation

Further input torque causes the next phalanges to turn until all of them wrap the object. In grasping operation the pin of distal phalanx remains at point $B$ of the upper link of the middle phalanx since no force is applied at the tip of distal phalanx. The position of the pin at point $\mathrm{B}$ lengthens the distance between point $\mathrm{C}$ and distal phalanx's pin so that grasping configuration is realized as illustrated in Fig. 5.

The extension of the finger after pinching or grasping to return to its original configuration as in Fig. 3 is provided by the spring. The mechanical limit prevents the finger mechanism from over extension.

Geometric optimization: The behaviour of an underactuated bar linkage type underactuated finger such as its grasping kinematics and force depends greatly on its geometry and spring stiffness parameters (Laliberte and Gosselin, 1998). Not any combination of the linkage length can realize both grasping and pinching configuration. Therefore, this study focuses on the geometrical parameter optimization of the mechanism so that it can reach pinching and grasping kinematics as human finger. Considering this factor, the optimization procedure is formulated to minimize the following objective functions:

- Pinching kinematics optimization

- Grasping kinematics optimization

Design variable and optimization criteria: The design variables to be optimized are the length of each linkage that builds up the finger, which are $1_{1}, 1_{2}, 1_{3}, 1_{4}$, $1_{5}, 1_{6}, 1_{7}, 1_{8}, 1_{9}$ as shown in Fig. 6 . Since the link with length $l_{6}$ need to be short during pinching and long during grasping, it is divided into $1_{6 \mathrm{p}}$ and $1_{6 \mathrm{~g}}$ in the optimization problem. 


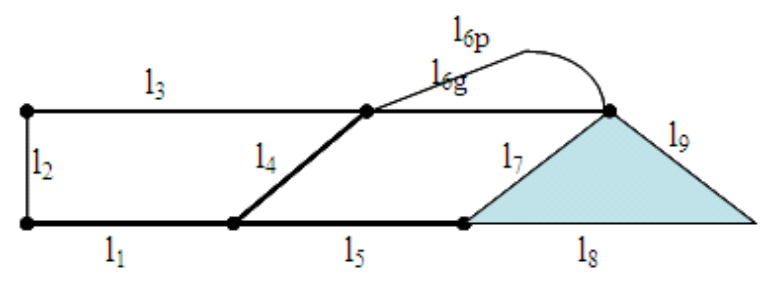

Fig. 6: Design variables

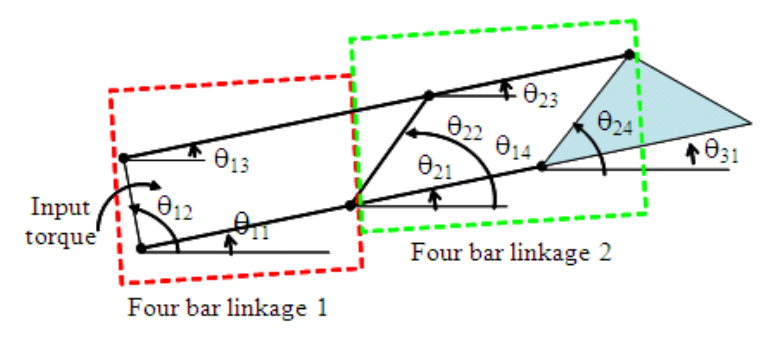

Fig. 7: Two four bar linkages

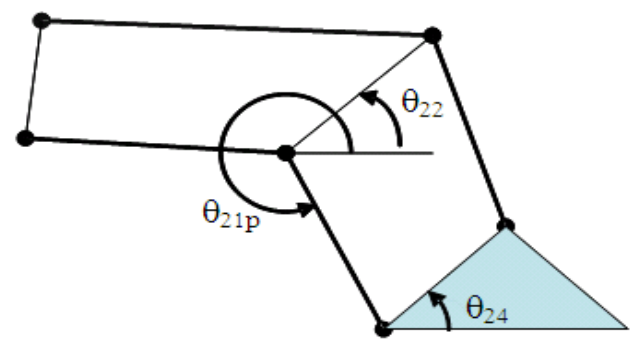

Fig. 8: Under actuated finger in pinching configuration

Pinching kinematics optimization: In pinching kinematics optimization problem, the designed finger mechanism is considered as two sets of four bar linkage where the first set is made of linkages with length $1_{1}, 1_{2}, 1_{3}, 1_{4}$ and the bars of $1_{4}, 1_{5}, 1_{6}, 1_{7}$ long are regarded as the second set, as shown in Fig. 7.

When the input torque in clockwise direction is applied on link with length $l_{2}$, the mechanism will move as one unit until the distal phalange touches the object. The triangular bar turns counter clockwise when the surface of the distal phalange touches the ground and this causes the link with length $1_{5}$ to be more vertical.

Only linkages with the length $1_{4}, 1_{5}, 1_{6}$ and $1_{7}$ as shown in Fig. 8 are considered in the pinching kinematics formulation. In this case, the linkage with length $l_{4}$ acts as the base and linkage $l_{7}$ is the driving link. At this base angle $\theta_{22}$, the output angle $\theta_{21 \mathrm{p}}$ can be calculated using the four bar linkage kinematics as:

$\theta_{21 \mathrm{p}}=\pi+2 \tan ^{-1}\left[\frac{-\mathrm{B}_{\mathrm{p}} \pm \sqrt{\mathrm{B}_{\mathrm{p}}{ }^{2}-\mathrm{C}_{\mathrm{p}}{ }^{2}+\mathrm{A}_{\mathrm{p}}{ }^{2}}}{\mathrm{C}_{\mathrm{p}}-\mathrm{A}_{\mathrm{p}}}\right]$
Where:

$$
\begin{aligned}
\mathrm{A}_{\mathrm{p}}= & 21_{4} 1_{5} \cos \left(\theta_{22}\right)-2 \mathrm{l}_{5} \mathrm{l}_{7} \cos \theta_{24} \\
\mathrm{~B}_{\mathrm{p}}= & 21_{4} 1_{5} \sin \left(\theta_{22}\right)-21_{5} 1_{7} \sin \theta_{24} \\
\mathrm{C}_{\mathrm{p}}= & 1_{4}^{2}+1_{5}^{2}+1_{7}^{2}-1_{6 \mathrm{p}}^{2}-21_{4} 1_{7} \\
& \left(\cos \theta_{22} \cos \theta_{24}+\sin \theta_{22} \sin \theta_{24}\right)
\end{aligned}
$$

The angle made by human finger during pinching $\theta_{21 \text { hp }}$ is measured from a photo taken and compared to the artificial finger's angle, $\theta_{21 \mathrm{p}}$ made during pinching. The objective function, $f_{1}$ has to minimize the difference between the angle made human finger and the artificial finger. This can be represented by the first objective function, $\mathrm{f}_{1}$ as:

$$
\mathrm{f}_{1}=\left|\theta_{21 \mathrm{hp}}-\theta_{21 \mathrm{p}}\right|
$$

Grasping kinematic optimization: Similar to pinching kinematics optimization, in grasping kinematics optimization, the bar linkage mechanism is also regarded as made of two sets of four bar linkage and the input torque is provided to the link with length $l_{2}$. Since the phalanges' movement are constrained by the object, two stages are considered in the optimization problem, which are when the only the proximal phalange touches an object and when both proximal and middle phalanges are in contact with the object.

When the proximal phalange touches an object in the first stage, the link with length $l_{1}$ can be regarded as the base and $\theta_{12}$ is the input angle. The output angle of the first for bar linkage, $\theta_{14}$ is equivalent to the input angle for the second four bar linkage, $\theta_{22}$. The value of $\theta_{14}$ and the value of the output angle of the second four bar linkage $\theta_{24}$ can be calculated as:

$\theta_{i 4}=2 \tan ^{-1}\left[\frac{-B_{i} \pm \sqrt{B_{i}^{2}-C_{i}^{2}+A_{i}^{2}}}{C_{i}-A_{i}}\right], i=1,2$

Where:

$$
\begin{aligned}
\mathrm{A}_{2}= & 21_{5} 1_{7} \cos \theta_{21}-21_{5} 1_{7} \cos \theta_{22} \\
\mathrm{~B}_{1}= & 21_{1} 1_{4} \sin \theta_{11}-21_{2} 1_{4} \sin \theta_{12} \\
\mathrm{~B}_{2}= & 21_{5} 1_{7} \sin \theta_{21}-21_{5} 1_{7} \sin \theta_{22} \\
\mathrm{~A}_{1}= & 21_{1} 1_{4} \cos \theta_{11}-21_{2} 1_{4} \cos \theta_{12} \\
\mathrm{C}_{1}= & 1_{1}^{2}+1_{2}^{2}+1_{4}^{2}-1_{3}^{2}-21_{2} 1_{4} \\
& \left(\cos \theta_{11} \cos \theta_{12}+\sin \theta_{11} \sin \theta_{12}\right) \\
\mathrm{C}_{2}= & 1_{5}^{2}+l_{4}^{2}+1_{7}^{2}-1_{6 \mathrm{~g}}^{2}-21_{4} 1_{7} \\
& \left(\cos \theta_{21} \cos \theta_{22}+\sin \theta_{21} \sin \theta_{22}\right)
\end{aligned}
$$


$i$ is the four bar linkage number.

From (4), the angle $\theta_{21 \mathrm{~g}}$ before middle phalange touches the object can be calculated as:

$$
\theta_{21 \mathrm{~g}}=\theta_{14}-\left[\pi-\tan ^{-1}\left(\frac{1_{2}}{l_{1}}\right)-\cos ^{-1}\left(1_{3}^{2}-\frac{1_{4}^{2}-\left(1_{1}^{2}+l_{2}^{2}\right)}{-21_{4} \sqrt{1_{1}^{2}+l_{2}^{2}}}\right)\right]
$$

and the value of $\theta_{31 \mathrm{~g}}$ after both proximal and middle phalanges have wrapped the object can be calculated as:

$$
\theta_{31 \mathrm{~g}}=\theta_{24}-\cos ^{-1}\left(\frac{1_{9}^{2}-l_{7}^{2}-l_{8}^{2}}{-21_{7} 1_{8}}\right)
$$

The corresponding angles that human's middle phalange, $\theta_{21 \mathrm{hg}}$ and distal phalange and $\theta_{31 \mathrm{hg}}$ during grasping are measured from a photo of human finger performing grasping operation and compared with the angles made by the robotic finger. The objective function in this case, $f_{2}$ has to minimize the difference between the angles mage by human and the artificial finger during grasping, which can be mathematically described as:

$$
\mathrm{f}_{2}=\left|\theta_{2 \operatorname{lng}}-\theta_{21 \mathrm{~g}}\right|+\left|\theta_{31 \mathrm{hg}}-\theta_{31 \mathrm{~g}}\right|
$$

Constraints: The optimization problem is subjected to the constraints which are due to finger size and also the four bar linkage kinematics equation.

In terms finger size, the length, $\mathrm{L}$ and thickness, $\mathrm{T}$ of the artificial finger has to be as close as possible to human's size, $L_{h}$ and $T_{h}$, which can be represented as:

$$
\mathrm{L}=\mathrm{L}_{\mathrm{h}}
$$

$\mathrm{T}=\mathrm{T}_{\mathrm{h}}$

Where:

$$
\mathrm{L}=\mathrm{l}_{1}+\mathrm{l}_{5}+\mathrm{l}_{8}
$$

$\mathrm{T}=1_{2}$

Considering the kinematics equation of the four bar linkage in (1) and (4), the optimization problem is also subjected to the following constraint, so that the solutions to these equations exist and the mechanism can be assembled in the specified position:

$$
\begin{aligned}
& \mathrm{B}_{\mathrm{p}}{ }^{2}-\mathrm{C}_{\mathrm{p}}{ }^{2}+\mathrm{A}_{\mathrm{p}}{ }^{2}>0 \\
& \mathrm{~B}_{\mathrm{i}}{ }^{2}-\mathrm{C}_{\mathrm{i}}{ }^{2}+\mathrm{A}_{\mathrm{i}}{ }^{2}>0 \quad \mathrm{i}=1,2
\end{aligned}
$$

Optimization formulation: The optimization problem has to minimize the multiple objective function:

$\mathrm{F}=\mathrm{w}_{1} \mathrm{f}_{1}+\mathrm{w}_{2} \mathrm{f}_{2}$

where, $\mathrm{w}_{\mathrm{i}}, \mathrm{i}=1,2$ are the weight coefficients and subjected to the constraints:

$\mathrm{g}_{1}=\left|\mathrm{L}-\mathrm{L}_{\mathrm{h}}\right|<0$

$\mathrm{g}_{2}=\left|\mathrm{T}-\mathrm{T}_{\mathrm{h}}\right|<0$

$\mathrm{g}_{3}=-\mathrm{B}_{\mathrm{i}}{ }^{2}+\mathrm{C}_{\mathrm{i}}^{2}-\mathrm{A}_{\mathrm{i}}^{2}<0 \quad \mathrm{i}=1,2$

$\mathrm{g}_{4}=-\mathrm{B}_{\mathrm{p}}{ }^{2}+\mathrm{C}_{\mathrm{p}}{ }^{2}-\mathrm{A}_{\mathrm{p}}{ }^{2}<0$

The optimization algorithm was carried out using the Sequential Quadratic programming in the Matlab Optimization Toolbox.

\section{RESULTS}

A set of initial lengths was chosen and the weight coefficients were set as 1 . The lower and upper boundaries of each length were selected as in Table 1 . The resulting optimized parameters were summarized in Table 2. The solution was obtained by convergence of local optimum. From the results it can be seen that the optimized parameter set reduces the error in fulfilling the pinching and grasping kinematics criteria.

Table 1: Upper and lower boundaries

\begin{tabular}{lll}
\hline Length & $\begin{array}{l}\text { Upper boundary } \\
(\mathrm{mm})\end{array}$ & $\begin{array}{l}\text { Lower boundary } \\
(\mathrm{mm})\end{array}$ \\
\hline$l_{1}$ & 10.0 & 30.0 \\
$l_{2}$ & 10.0 & 20.0 \\
$l_{3}$ & 10.0 & 40.0 \\
$l_{4}$ & 10.0 & 20.0 \\
$l_{5}$ & 10.0 & 30.0 \\
$l_{6 \mathrm{p}}$ & 10.0 & 30.0 \\
$l_{6 \mathrm{~g}}$ & 10.0 & 30.0 \\
$l_{7}$ & 10.0 & 20.0 \\
$l_{8}$ & 10.0 & 30.0 \\
$l_{9}$ & 10.0 & 20.0 \\
\hline
\end{tabular}

Table 2: Optimized solution and initial guess

\begin{tabular}{lll}
\hline Length & $\begin{array}{l}\text { Initial guess } \\
(\mathrm{mm})\end{array}$ & $\begin{array}{l}\text { Optimized solution } \\
(\mathrm{mm})\end{array}$ \\
\hline$l_{1}$ & 25.0 & 25.0 \\
$l_{2}$ & 18.0 & 18.3 \\
$l_{3}$ & 35.0 & 34.6 \\
$l_{4}$ & 14.0 & 13.9 \\
$l_{5}$ & 21.0 & 22.5 \\
$l_{6 \mathrm{p}}$ & 25.0 & 22.5 \\
$l_{6 \mathrm{~g}}$ & 29.0 & 29.8 \\
$l_{7}$ & 18.0 & 17.6 \\
$l_{8}$ & 24.0 & 25.3 \\
$l_{9}$ & 11.0 & 12.5 \\
Error due to pinching $(\mathrm{rad})$ & $8.0 \times 10^{-1}$ & $0.2 \times 10^{-6}$ \\
Error due to grasping (rad) & $5.5 \times 10^{-1}$ & $1.5 \times 10^{-6}$ \\
Total error (rad) & 1.35 & $1.7 \times 10^{-6}$ \\
\hline
\end{tabular}




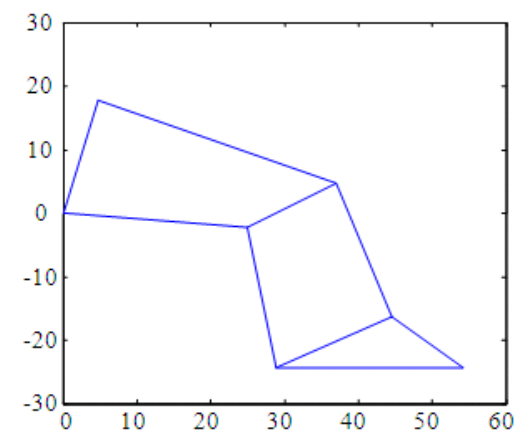

Fig. 9: Artificial finger with optimized length in pinching position

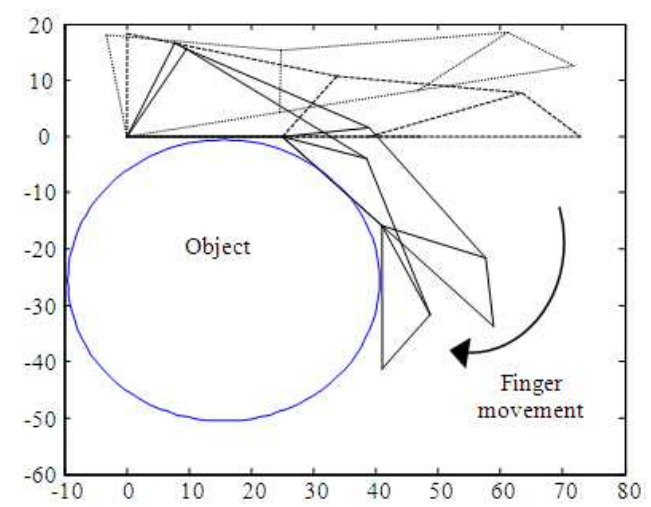

Fig. 10: Artificial finger with optimized length encircling a cylindrical object

Figure 9 and 10 illustrate the pinching and grasping of the artificial finger with optimized lengths around an object respectively. The arrow indicates the direction of the finger movement.

\section{DISCUSSION}

The result provides an optimized the underactuated bar linkage lengths to achieve pinching and grasping operation. It gives a better parameter set as it results in much smaller error compared to the initial guess. These values will be used in fabricating the finger mechanism in the future.

\section{CONCLUSION}

A new underactuated finger mechanism that resembles the human finger to perform both pinching and grasping operation is presented in this study. The mechanism is made of seven bar linkages with a guiding slot in middle phalange link so that its length between PIP and DIP joints can be adjusted to achieve human finger's configuration during these two operations. Using the evaluation criteria for human finger kinematics, the length of the linkages is then optimized. The numerical result gives an improved set of geometrical parameters to achieve both of the configurations as it provides less error than the initial guess.

\section{REFERENCES}

Bekey, G.A., R. Tomovic and I. Zeljkovic, 1990. Control architecture for the Belgrade/ USC hand. Dextrous Robot Hands, Springer-Verlag, New York, USA., pp: 136-149.

Jacobsen, S.C., E.K. Iversen, D.F. Knutti, R.T. Johnson and K.B. Biggers, 1986. Design of the UTAH/M.I.T. dextrous hand. Proceeding of the IEEE International Conference on Robotics and Automation, Apr. 1986, IEEE Xplore Press, USA., pp: 1520-1532. DOI: 10.1109/ROBOT.1986.1087395

Laliberte, T. and C.M. Gosselin, 1998. Simulation and design of underactuated mechanical hands. Mech. Man Theory, 33: 39-57. DOI: 10.1016/S0094114X(97)00020-7.

Laliberte, T. and C.M. Gosselin, 2001. Underactuation in space robotic hands. Proceeding of the 6th International Symposium on Artificial Intelligence and Robotics and Automation in Space, June 18-22, Canadian Space Agency, St-Hubert, Quebec, Canada, pp: 1-8. http://robotics.estec.esa.int/iSAIRAS/isairas2001/ papers/Paper_AM025.pdf

Salisbury, J.K. and J.J. Craig, 1982. Articulated hands: Force control and kinematics issues. Int. J. Robot. Res., 1: 4-17. DOI: 10.1177/027836498200100102.

Zollo, L., S. Rocecella, R. Tucci, B. Siciliano and E. Guglielmelli et al., 2007. Biomechatronic design and control of an anthropomorphic artificial hand for prosthetic and robotic applications. IEEE/ASME Trans. Mechatron., 12: 418-429. http://ieeexplore.iee.org/stamp/stamp.jsp?tp=\&arn umber $=1639121 \&$ userType $=\& \operatorname{tag}=1$ 